\title{
Relationship between Hypogonadal Symptoms, Sexual Dysfunction and Chronic Prostatitis in Middle-Aged Men by Self-Reported Questionnaires, even without Biochemical Testosterone Deficiency
}

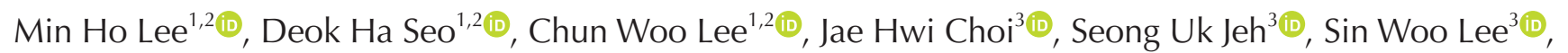
See Min $\mathrm{Choi}^{3}$ (D), Jeong Seok Hwa ${ }^{3}$, Jae Seog Hyun ${ }^{3}$, Ky Hyun Chung ${ }^{3}$ (D) Sung Chul Kam ${ }^{1,2}$

${ }^{1}$ Department of Urology, Gyeongsang National University Changwon Hospital, Changwon, ${ }^{2}$ Department of Urology, Institute of Health Sciences, Gyeongsang National University College of Medicine, ${ }^{3}$ Department of Urology, Gyeongsang National University Hospital, Gyeongsang National University School of Medicine, Jinju, Korea

Purpose: To investigate the association of erectile dysfunction (ED), premature ejaculation (PE), and chronic prostatitis/chronic pelvic pain syndrome (CP/CPPS) in men with late-onset hypogonadism ( $\mathrm{LOH})$.

Materials and Methods: We reviewed the data of 408 enrolled men between January 2014 and January 2019. All participants completed the Androgen Deficiency in the Aging Male (ADAM), international index of erectile function-5 (IIEF-5), National Institutes of Health chronic prostatitis symptom index (NIH-CPSI), and premature ejaculation diagnostic tool (PEDT) questionnaires. Participants were divided by ADAM positive (ADAM+: Group 1) and ADAM negative (ADAM-: Group 2).

Results: Total of 289 subjects were in Group 1 and 119 were in Group 2. The mean age was 53.8 \pm 7.8 years. The mean total testosterone was $4.8 \pm 1.2 \mathrm{ng} / \mathrm{dL}$ and showed no differences between the groups $(p=0.839)$. In Groups 1 and 2, ED (IIEF $\leq 21)$ was identified in $233(80.6 \%)$ versus $37(31.1 \%)$, respectively $(p<0.001)$. The prevalence of PE (PEDT $\geq 9)$ was $112(38.7 \%)$ versus $13(10.9 \%)$ in Groups 1 and 2, respectively $(p<0.001)$. However, $\mathrm{PE}$ (intravaginal ejaculation latency time $<5$ minutes) showed no differences between the groups $(\mathrm{p}=0.863)$. The incidence of chronic prostatitis (NIH-CPSI pain score $\geq 4)$ showed significant differences with $49(17.0 \%)$ versus $8(6.7 \%)$ in Groups 1 and 2 , respectively $(\mathrm{p}=0.007)$. IIEF-5 total score showed the significantly highest negative correlation $(r=-0.313, p<0.001)$.

Conclusions: Those who complained of $\mathrm{LOH}$ symptoms and positive results in the ADAM questionnaire need to be assessed concurrently with the above questionnaires. This could aid useful to detect of ED, PE, and chronic prostatitis co-occurrence.

Keywords: Erectile dysfunction; Hypogonadism; Premature ejaculation; Prostatitis; Questionnaires

This is an Open Access article distributed under the terms of the Creative Commons Attribution Non-Commercial License (http://creativecommons.org/licenses/by-nc/4.0) which permits unrestricted non-commercial use, distribution, and reproduction in any medium, provided the original work is properly cited.

Received: Sep 18, 2019 Revised: Jan 15, 2020 Accepted: Jan 29, 2020 Published online Feb 14, 2020

Correspondence to: Sung Chul Kam iD https://orcid.org/0000-0001-5403-3623

Department of Urology, Gyeongsang National University Changwon Hospital, Gyeongsang National University School of Medicine, 11 Samjeongja-ro, Seongsan-gu, Changwon 51472, Korea.

Tel: +82-55-214-3876, Fax: +82-55-214-3264, E-mail: kamsungchul@hanmail.net 


\section{INTRODUCTION}

The National Health and Social Life Survey and Massachusetts Male Aging Study reported that the prevalence of erectile dysfunction (ED) increases with age [1,2]. Additionally, the incidence increases with several comorbidities, including hypertension, diabetes mellitus, and coronary heart disease [3]. Late-onset hypogonadism $(\mathrm{LOH})$, which is characterized by decreased sexual function and desire, also correlates with ED.

Globally, premature ejaculation (PE) is one of the most common sexual dysfunction (SD) in men; the prevalence of $\mathrm{PE}$ is approximately $14.0 \%$ to $19.5 \%$ [4]. $\mathrm{PE}$ affects not only the patients but also causes considerable sexual distress in their partners. PE has a multifactorial pathogenesis, including psychologic and organic factors. Previous studies have reported that apart from the imbalance of serotonin receptors, age, lower urinary tract symptoms (LUTS), ED, prostatitis, and LOH may also contribute to occurrence of PE [5-7].

Chronic prostatitis/chronic pelvic pain syndrome (CP/CPPS), defined as National Institutes of Health (NIH) category III prostatitis, is a common syndrome affecting about $15 \%$ of all men [8]. Tran and Shoskes [9] reviewed the association of CP/CPPS and SD, which revealed that men with $\mathrm{CP} / \mathrm{CPPS}$ were significantly more likely to experience ED and PE than the general population.

In a previous study, biochemical androgen deficiency (bAD) was a common finding in men with ED. It has been postulated that sex hormones may also be an important factor in the development of prostatitis [10]. Several studies have already investigated the association of ED, PE and CP/CPPS or ED, PE and LOH, respectively [11-13]. Morley et al [14] evaluated the validation of screening LOH using the Androgen Deficiency in the Aging Male (ADAM) questionnaire, and it showed acceptable data in their study. Kang et al [15] also studied SD and LOH using the ADAM questionnaire, but the authors only revealed their risk factors.

And none of the previous study revealed the association of SD, CP in men who complain of hypogonadal symptoms. In the present study, we investigated the association of ED, PE, and CP/CPPS in men with $\mathrm{LOH}$ using simple self-reported questionnaires, which are the preferred screening tool for non-invasive method with reliable results.

\section{MATERIALS AND METHODS}

\section{Study population}

We reviewed the data of 1,120 men who visited our health care center for check-up of their health status between January 2014 and January 2019. So, all participants were evaluated by a clinical assessment, weight, height, body mass index (BMI), and other characteristics. Total testosterone (TT), total cholesterol, triglycerides (TG), high density-lipoprotein, low densitylipoprotein, glycosylated hemoglobin, blood counts, and prostate specific antigen were assessed from morning blood samples in most of the participants to minimize diurnal fluctuations. Exclusion criteria: (1) The participants who were younger than 40 years and older than 79 years because we focused on middle-aged men. (2) Those who did not completely fill out all four questionnaires. (3) Subjects pre-diagnosed and medication with hypertension, diabetes-mellitus, cerebrovascular disease, coronary heart disease, psychologic disease, neurogenic disease, $\mathrm{LOH}$, previous urologic surgical history, recent urologic infection history. (4) Those who refused to take transrectal ultrasonography and urine flowmetry. A total of 408 participants were enrolled.

\section{Ethics statement}

The present study protocol was reviewed and approved by the Institutional Review Board (IRB) of Gyeongsang National University Hospital (IRB No. 2019-10-010). The need for informed consent was waived by the IRB based on the retrospective nature of the study.

\section{Questionnaire}

All participants completed the ADAM, international index of erectile function-5 (IIEF-5), NIH-chronic prostatitis symptom index (NIH-CPSI), and premature ejaculation diagnostic tool (PEDT) questionnaires, which have validated Korean versions, after sufficient explanation of each item. Participants also reported the intravaginal ejaculation latency time (IELT). ADAM positive (ADAM+: Group 1) participants were defined as: (1) those who answered yes for question 1 or 7; or (2) a summed total score of more than 3 in the remaining eight questions. ADAM negative (ADAM-) participants were classified as Group 2. NIH-CPSI pain scores $\geq 4$ were defined as CP positive and NIH-CPSI pain scores $<4$ were defined as CP negative. Partici- 
pants reported their IELT: $<1$ minute, $1-5$ minutes, and $>5$ minutes. IELT was defined as PE $>5$ minutes. Men with PEDT scores $\geq 9$ were classified as having PE. We classified IIEF-5 scores $\leq 21$ as having ED.

\section{Statistical analysis}

Data are expressed as mean value and standard deviation unless otherwise specified. Continuous variables were evaluated using the Student's t-test and categorical variables were evaluated using the chi-square test for intergroup comparisons. The correlations and statistical analyses were performed using IBM SPSS ver. 25.0 (IBM Corp., Armonk, NY, USA). All tests were two-sided and significant differences were set at $\mathrm{p}<0.05$.

\section{RESULTS}

Table 1 shows the characteristics of the 408 participants and those of Group 1 (ADAM+) and Group 2 (ADAM-). The mean age was $53.8 \pm 7.8$ years. Age, weight, height, and BMI showed no significant differences between the groups. Except for TG ( $p=0.035)$, all other results from blood samples showed no differences between the groups. Although all participants had not provided blood samples early in the morning, the mean
TT was $4.8 \pm 1.2 \mathrm{ng} / \mathrm{dL}$ and showed no significant differences between the groups $(p=0.839)$. The prostate size measured by transrectal ultrasonography and the max flow rate by urine flowmetry showed similar results ( $\mathrm{p}=0.641$ and $\mathrm{p}=0.685$, respectively). A total of 11 subjects reported their medical history, such as hypertension, diabetes mellitus, and coronary heart disease.

Table 2 shows the sub-scores of NIH-CPIS, IIEF-5, and PEDT according to the groups. The mean of NIHCPSI total, pain, voiding, quality of life (QOL) scores were $9.1 \pm 6.5,1.7 \pm 2.4,2.9 \pm 2.3$, and $4.3 \pm 2.4$, respectively, in Group 1. All scores were significantly higher in Group 1 than in Group 2 ( $p<0.001)$. The mean of $\Pi E F$ 5 total and satisfaction scores were $16.9 \pm 5.5$ and $1.7 \pm 0.8$, respectively, which were lower than those of Group 2 $(p<0.001)$. The mean of PEDT score was significantly higher in Group 1 than in Group $2(p<0.001)$.

Table 3 shows the comparison of the prevalence and severity of $\mathrm{ED}, \mathrm{PE}$, and $\mathrm{CP}$ according to the groups. In Groups 1 and 2, ED ( $\Pi E F \leq 21)$ was identified in 233 (80.6\%) versus 37 (31.1\%); moderate and severe ED was only found in Group 1. These results showed significant differences $(\mathrm{p}<0.001)$. The prevalence of $\mathrm{PE}(\mathrm{PEDT} \geq 9)$ was 112 (38.7\%) versus $13(10.9 \%)$ in Groups 1 and 2, respectively $(p<0.001)$. However, the incidence of $\mathrm{PE}$

Table 1. Baseline characteristics of all participants

\begin{tabular}{|c|c|c|c|c|}
\hline Variable & Total $(n=408)$ & Group $1(n=289)($ ADAM +$)$ & Group 2 ( $n=119)$ (ADAM-) & p-value \\
\hline Age (y) & $53.8 \pm 7.8$ & $53.9 \pm 8.0$ & $53.3 \pm 7.3$ & 0.486 \\
\hline Height (cm) & $171.0 \pm 5.9$ & $171.1 \pm 5.8$ & $170.7 \pm 5.7$ & 0.573 \\
\hline Weight $(\mathrm{kg})$ & $72.2 \pm 9.4$ & $72.6 \pm 9.5$ & $71.2 \pm 9.2$ & 0.181 \\
\hline Body mass index $\left(\mathrm{kg} / \mathrm{m}^{2}\right)$ & $24.6 \pm 2.8$ & $24.7 \pm 2.7$ & $24.4 \pm 3.0$ & 0.356 \\
\hline WBC $\left(\times 10^{3}\right)$ & $6.1 \pm 1.3$ & $6.1 \pm 1.7$ & $6.0 \pm 1.9$ & 0.893 \\
\hline Hemoglobin (g/L) & $15.2 \pm 1.0$ & $15.2 \pm 0.9$ & $15.2 \pm 1.1$ & 0.794 \\
\hline Cholesterol (mg/dL) & $185.8 \pm 35.5$ & $185.2 \pm 35.6$ & $187.1 \pm 35.6$ & 0.615 \\
\hline Triglycerides (mg/dL) & $136.7 \pm 82.6$ & $130.2 \pm 70.9$ & $152.5 \pm 104.6$ & 0.035 \\
\hline $\mathrm{HDL}(\mathrm{mg} / \mathrm{dL})$ & $52.0 \pm 13.1$ & $51.9 \pm 13.1$ & $52.2 \pm 13.3$ & 0.839 \\
\hline $\mathrm{LDL}(\mathrm{mg} / \mathrm{dL})$ & $122.2 \pm 33.1$ & $122.4 \pm 33.7$ & $121.8 \pm 31.5$ & 0.869 \\
\hline Protein (g/dL) & $7.2 \pm 0.4$ & $7.2 \pm 0.4$ & $7.1 \pm 0.4$ & 0.185 \\
\hline Albumin (g/dL) & $4.6 \pm 0.2$ & $4.6 \pm 0.3$ & $4.5 \pm 0.2$ & 0.332 \\
\hline C-reactive protein (mg/L) & $1.1 \pm 1.4$ & $1.1 \pm 1.3$ & $1.0 \pm 1.4$ & 0.757 \\
\hline $\operatorname{HbA1c}(\%)$ & $5.7 \pm 0.7$ & $5.7 \pm 0.7$ & $5.8 \pm 0.8$ & 0.350 \\
\hline Total testosterone $(\mathrm{ng} / \mathrm{mL})$ & $4.8 \pm 1.2$ & $4.7 \pm 1.7$ & $4.8 \pm 1.3$ & 0.839 \\
\hline Total PSA (ng/mL) & $1.2 \pm 1.0$ & $1.2 \pm 0.9$ & $1.1 \pm 0.8$ & 0.287 \\
\hline Prostate size (g) & $27.5 \pm 8.5$ & $27.6 \pm 8.3$ & $27.2 \pm 9.1$ & 0.641 \\
\hline Maximum flow rate $(\mathrm{mL} / \mathrm{s})$ & $17.8 \pm 8.8$ & $17.9 \pm 8.9$ & $17.5 \pm 8.8$ & 0.685 \\
\hline
\end{tabular}

Values are presents as mean \pm standard deviation.

ADAM: Androgen Deficiency in the Aging Male, WBC: white blood cell, HDL: high density-lipoprotein, LDL: low density-lipoprotein, HbA1c: glycosylated hemoglobin, PSA: prostate specific antigen. 
(IELT $<5$ minutes) showed no differences between the groups $(\mathrm{p}=0.863)$. Originally, we collected data of IELT for $<1$ minute, $1-5$ minutes, and $>5$ minutes, and these subclassifications might have induced these insignificant differences. The incidence of CP (NIH-CPSI pain score $\geq 4)$ showed significant differences with $49(17.0 \%)$ versus 8 (6.7\%) in Groups 1 and 2 , respectively $(\mathrm{p}=0.007)$. Beyond these results, more ED, PE, and $\mathrm{CP}$ patients were identified as ADAM+.

Table 4 shows the Pearson's correlation between the ADAM questionnaire and NIH-CPSI, IIEF-5, and PEDT questionnaire scores. IIEF-5 total score showed the significantly highest negative correlation $(\mathrm{r}=-0.313$, $\mathrm{p}<0.001)$. NIH-CPSI total score $(\mathrm{r}=0.256)$, pain score

Table 2. The comparisons of the self-reported questionnaire scores according to ADAM

\begin{tabular}{lccc}
\hline Variable & $\begin{array}{c}\text { Group } 1(\mathrm{n}=289) \\
\text { (ADAM+) }\end{array}$ & $\begin{array}{c}\text { Group 2 }(\mathrm{n}=119) \\
\text { (ADAM-) }\end{array}$ & p-value \\
\hline IIEF-5 & $16.9 \pm 5.5$ & $22.6 \pm 2.4$ & $<0.001$ \\
PEDT & $7.6 \pm 4.1$ & $4.5 \pm 3.6$ & $<0.001$ \\
NIH-CPSI & & & \\
Pain & $1.7 \pm 2.4$ & $0.5 \pm 1.6$ & $<0.001$ \\
Voiding & $2.9 \pm 2.3$ & $1.5 \pm 1.5$ & $<0.001$ \\
QOL & $4.3 \pm 2.4$ & $3.1 \pm 1.9$ & $<0.001$ \\
Total & $9.1 \pm 6.5$ & $5.1 \pm 3.5$ & $<0.001$ \\
\hline
\end{tabular}

Values are presented as mean \pm standard deviation.

ADAM: Androgen Deficiency in the Aging Male, IIEF-5: international index of erection function-5, PEDT: premature ejaculation diagnostic tool, NIH-CPSI: National Institutes of Health chronic prostatitis symptom index, QOL: quality of life. $(\mathrm{r}=0.241)$, voiding score $(\mathrm{r}=0.257)$, and QOL score $(\mathrm{r}=0.233)$ also showed significant correlations (all $\mathrm{p}<0.001$ ). Additionally, PEDT total score showed a similar result $(r=0.215, p<0.001)$.

\section{DISCUSSION}

The present study was a cross-sectional study of the association between $\mathrm{ED}, \mathrm{PE}$, and $\mathrm{CP}$ among the men who had LOH symptoms based on the self-reported ADAM questionnaire. According to the European Male Ageing Study (EMAS), when the TT levels was less than $10 \mathrm{nM}$, the prevalence of bAD was $23.3 \%$ in men aged 40 to 79 years [16]. Moreover, the EMAS recently defined strict diagnostic criteria for $\mathrm{LOH}$ to include

Table 4. Correlation between ADAM and NIH-CPSI sub-scores, IIEF-5, PEDT with Pearson's correlation in ADAM+ group

\begin{tabular}{lcc}
\hline \multirow{2}{*}{ Variable } & \multicolumn{2}{c}{ Group $1(\mathrm{n}=289)($ ADAM +$)$} \\
\cline { 2 - 3 } & $\mathrm{r}$ & $\mathrm{p}$-value \\
\hline NIH-CPSI pain & 0.241 & $<0.001$ \\
NIH-CPSI voiding & 0.257 & $<0.001$ \\
NIH-CPSI QOL & 0.233 & $<0.001$ \\
NIH-CPSI total & 0.256 & $<0.001$ \\
IIEF-5 & -0.313 & $<0.001$ \\
PEDT & 0.215 & $<0.001$ \\
\hline
\end{tabular}

ADAM: Androgen Deficiency in the Aging Male, NIH-CPSI: National Institutes of Health chronic prostatitis symptom index, IIEF-5: international index of erection function-5, PEDT: premature ejaculation diagnostic tool, QOL: quality of life.

Table 3. Stratification of ED, PE, and CP according to ADAM

\begin{tabular}{|c|c|c|c|c|}
\hline Variable & Total $(n=408)$ & Group $1(n=289)(A D A M+)$ & Group 2 ( $n=119)$ (ADAM-) & $\mathrm{p}$-value \\
\hline $\mathrm{ED}(\mathrm{IIEF}-5 \leq 21)$ & $270(66.2)$ & $233(80.6)$ & $37(31.1)$ & $<0.001$ \\
\hline Mild (12-21) & $224(54.9)$ & $187(64.7)$ & $37(31.1)$ & \\
\hline Moderate (8-11) & $18(4.4)$ & $18(6.2)$ & $0(0)$ & \\
\hline Severe $(\leq 7)$ & $28(6.9)$ & $28(9.7)$ & $0(0)$ & \\
\hline PE (PEDT $\geq 9)$ & $125(30.6)$ & $112(38.7)$ & $13(10.9)$ & $<0.001$ \\
\hline Probable (9-10) & $58(14.2)$ & $51(17.6)$ & $7(5.9)$ & \\
\hline Definite $(\geq 11)$ & $67(16.4)$ & $61(21.1)$ & $6(5.0)$ & \\
\hline $\mathrm{PE}(\mathrm{IELT}<5 \mathrm{~min})$ & $226(55.3)$ & $161(55.7)$ & $65(54.6)$ & 0.863 \\
\hline Mild (1-5 min) & $194(47.5)$ & $137(47.4)$ & $57(47.9)$ & \\
\hline Severe $(<1 \mathrm{~min})$ & $32(7.8)$ & $24(8.3)$ & $8(6.7)$ & \\
\hline $\mathrm{CP}(\mathrm{NIH}-\mathrm{CPSI}$ pain score $\geq 4)$ & $57(14.0)$ & $49(17.0)$ & $8(6.7)$ & 0.007 \\
\hline
\end{tabular}

Values are presented as number (\%).

ED: erectile dysfunction, PE: premature ejaculation, CP: chronic prostatitis, ADAM: Androgen Deficiency in the Aging Male, IIEF-5: international index of erection function-5, PEDT: premature ejaculation diagnostic tool, IELT: intravaginal ejaculation latency time, NIH-CPSI: National Institutes of Health chronic prostatitis symptom index. 
the simultaneous presence of reproducibly low serum testosterone ( $\mathrm{TT}<11 \mathrm{nM}$ and free testosterone $<220 \mathrm{pM}$ ) and three sexual symptoms (ED, reduced libido, and morning erection). According to these criteria, only $2 \%$ of men aged 40 to 80 years have $\mathrm{LOH}$ [17].

In present study, despite non-repeated hormonal level check and the low number of participants, 54 participants (13.2\%) had LOH based on TT level (<300 ng/ $\mathrm{dL}$ ), which was consistent with overseas studies. On the other hand, Lee and Lee [18] reported that low TT level (cut-off value $<3.5 \mathrm{ng} / \mathrm{mL}$ ) was significantly correlated with prostatitis-like symptoms and cut-off value $<3.0$ $\mathrm{ng} / \mathrm{mL}$ showed no statistical differences. Originally, we assessed the association of ED, PE, and CP by the TT level. According to the TT level, only the prostate size and maximum flow rate showed significant differences between the $\mathrm{LOH}$ and normal participants $(\mathrm{p}=0.003$, $\mathrm{p}=0.049$, respectively). No other statistical differences were seen in any of the questionnaires. Lee et al [19] reported that TT showed no significant correlation with prostate size and Qmax in middle-aged eugonadal men $(\mathrm{TT} \geq 3.0 \mathrm{ng} / \mathrm{mL})$. However, we primarily focused on evaluate the relationship between urologic diseases according to the ADAM questionnaire groups.

Due to the retrospective nature of study and participants visiting our healthcare center for just a medical check-up, it was impossible to take the blood samples in the same time for all participants and difficult to perform repeat tests. Thus, we decided to assess the $\mathrm{LOH}$ group by using the ADAM questionnaire. The study by Tang et al [20] on ED in Chinese men with $\mathrm{LOH}$ was conducted using the ADAM/aging male's syndrome (AMS) questionnaires to screen participants aged 40 to 69 years. The rates of $\mathrm{LOH}$ were $80.77 \%$ and $32.34 \%$, the mean $\mathrm{AD}$ rates were $14.02 \%$ and $43.69 \%$ (according to the TT and cFT cut-off levels), and the clinical prevalence rates of $\mathrm{LOH}$ were $37.85 \%$ and $15.42 \%$ for $\mathrm{ADAM}+$ and AMS+ participants, respectively. Additionally, Marberger et al [21] found that $10.2 \%$ of the participants had $\mathrm{LOH}$, and in the present study, the clinical prevalence of $\mathrm{LOH}$ was $70.8 \%$ among enrolled men.

Lee [22] reported that PE is correlated with ED and LUTS in middle-aged men by using PEDT, IPSS and IIEF. The Odds ratio for PE also increased with the severity of LUTS or ED after adjusting for potential confounding factors, such as age, metabolic syndrome components, TT. However, to the best of our knowledge, this is the first study of association of $\mathrm{ED}$ and $\mathrm{PE}$, as well as $\mathrm{CP}$, in men with $\mathrm{LOH}$ symptoms by using the self-reported ADAM questionnaire. Previous studies postulated that ED is strongly related to metabolic syndrome and cardiovascular disease risk. Jackson et al [23] found that type 2 diabetes mellitus is the second strongest predictor of ED after age. Ogden et al [24] and others [25] showed that ED is more prevalent in men with metabolic syndrome, especially in obese patients. However, the present study did not assess whether the presence of ED was an effect of comorbidities or not, and all of the men were enrolled by the self-reported questionnaires. Additionally, the use of tobacco has positive predictive effect of worse ED. However, we also could not assess the social habits fully due to the retrospective nature of study.

Corona et al [26] reported that testosterone is known to have a central and peripheral action in the ejaculatory process and an epidemiological study in 2008 [7] reported that an increase in testosterone was associated with the prevalence of PE. By contrast, the attribution of ED as a result of hypogonadism needs to be debated. Marberger et al [21] refute the hypothesis that TT is an indicator of ED and stated that age is a more valuable predictor. Hypogonadism is a common finding in men with ED. The present study also indicated that the majority of men with $\mathrm{LOH}$ had $\mathrm{ED}$ (ADAM+; 233 [80.6\%] versus ADAM-; 37 [31.1\%], $\mathrm{p}<0.001)$. The severity of ED showed similar results. It has been reported that sex hormones may also be an important factor in the development of prostatitis [27]. Thus far, an evidence of a link between CP/CPPS and ED remains to be determined. Recent findings regarding the genetics of patients with $\mathrm{CP}$ suggest an underlying problem with androgen regulation that may contribute to the development of prostatitis [28]. In one small case-control study, patients with CP/CPPS were found to have higher serum levels of androstenedione and testosterone and lower levels of cortisol as compared to the controls [29]. Our study showed the same result as those of the above studies: the Group 1 had 49 participants (17.0\%) with CP, and the Group 2 had 8 participants (6.7\%) with $\mathrm{CP}(\mathrm{p}=0.007)$.

Although these results were analyzed by only selfreported questionnaires, the prevalence differences between groups revealed a useful information to clinicians. It is difficult to regard $\mathrm{ED}, \mathrm{PE}$, and $\mathrm{CP}$ as individual diseases in men with $\mathrm{LOH}$. Additionally, the 
statistical positive correlations between the ADAM questionnaire and NIH-CPSI sub-scores, IIEF-5 total score, and PEDT total score were also the power of the present study. Nevertheless, evaluation of correlation between the IIEF-5 and ADAM questionnaire can be controversial. Because who complain for question 1 or 7 of ADAM, they can be considered as ADAM+ and it will be somewhat redundant. But, ADAM+ include those who also complain of more than three other questions of $\mathrm{ADAM}$ and has normal erectile function. So we decided evaluate the correlation of IIEF-5 and ADAM questionnaire.

This study has some limitations. First, as mentioned above, the retrospective design and cross-sectional nature make inferences problematic. Second, the collected data were from a single institution and this indicates a potential selection bias; however, the sample size was relatively large to validate our results. We also collecting data continuously and multicenter study will be designed closely. Third, the TT level check was not done more than twice, and the existence of time differences may have caused non-significant findings in bAD groups. This will need the implementation of a more precise and consistent protocol in our future study. Lastly, the protocol of the study by self-reported questionnaires might create another limitation. Selfreported scores reflect the diagnosis of $\mathrm{LOH}, \mathrm{ED}, \mathrm{PE}$ and $\mathrm{CP}$ is non-sense to global guidelines. It also inaccurately than does biological and/or histological diagnosis. However, our results can be useful to primary clinicians to make suspicion for SD, CP in men who complain of LOH symptoms only by self-reported questionnaire, even it is not absolute diagnosis.

\section{CONCLUSIONS}

More ED, PE, and CP participants were found in the Group 1. Severe cases were also more common in the Group 1. All of the NIH-CPSI sub-scores, IIEF-5, and PEDT score showed positive correlations with ADAM questionnaire scores. This suggests that those who complained of LOH symptoms and positive results in the ADAM questionnaire need to be assessed concurrently with the above questionnaires. This could aid to screening of $\mathrm{ED}, \mathrm{PE}$, and $\mathrm{CP}$ co-occurrence.

\section{Conflict of Interest}

The authors have nothing to disclose.

\section{Author Contribution}

Conceptualization: MHL, SCK. Data curation: MHL, SCK. Formal analysis: MHL, SCK. Investigation: DHS, CWL, JHC, SUJ, SWL, SMC. Methodology: MHL, SCK. Project administration: MHL, SCK. Resources: JSH, JSH, KHC. Supervision: SCK. Validation: SCK, JSH. Writing - original draft: MHL. Writing review \& editing: MHL, SCK.

\section{Data Sharing Statement}

The data analyzed for this study have been deposited in HARVARD Dataverse and are available at https://doi. org/10.7910/DVN/EBLVAG.

\section{REFERENCES}

1. Laumann EO, Paik A, Rosen RC. The epidemiology of erectile dysfunction: results from the National Health and Social Life Survey. Int J Impot Res 1999;11 Suppl 1:S60-4.

2. Johannes CB, Araujo AB, Feldman HA, Derby CA, Kleinman $\mathrm{KP}, \mathrm{McKinlay}$ JB. Incidence of erectile dysfunction in men 40 to 69 years old: longitudinal results from the Massachusetts male aging study. J Urol 2000;163:460-3.

3. Wein AJ, Kavoussi LR, Partin AW, Peters CA. CampbellWalsh urology. 11th ed. Philadelphia: Elsevier Saunders; 2016;648-9

4. McMahon CG, Lee G, Park JK, Adaikan PG. Premature ejaculation and erectile dysfunction prevalence and attitudes in the Asia-Pacific region. J Sex Med 2012;9:454-65.

5. Wein AJ, Coyne KS, Tubaro A, Sexton CC, Kopp ZS, Aiyer LP. The impact of lower urinary tract symptoms on male sexual health: EpiLUTS. BJU Int 2009;103 Suppl 3:33-41.

6. Gonen M, Kalkan M, Cenker A, Ozkardes H. Prevalence of premature ejaculation in Turkish men with chronic pelvic pain syndrome. J Androl 2005;26:601-3.

7. Corona G, Jannini EA, Mannucci E, Fisher AD, Lotti F, Petrone L, et al. Different testosterone levels are associated with ejaculatory dysfunction. J Sex Med 2008;5:1991-8.

8. Schaeffer AJ, Datta NS, Fowler JE Jr, Krieger JN, Litwin MS, Nadler RB, et al. Overview summary statement. Diagnosis and management of chronic prostatitis/chronic pelvic pain syndrome (CP/CPPS). Urology 2002;60(6 Suppl):1-4.

9. Tran CN, Shoskes DA. Sexual dysfunction in chronic prosta- 
titis/chronic pelvic pain syndrome. World J Urol 2013;31:7416.

10. Pontari MA, Ruggieri MR. Mechanisms in prostatitis/chronic pelvic pain syndrome. J Urol 2008;179:S61-7.

11. Yassin AA, Nettleship JE, Almehmadi Y, Yassin DJ, El Douaihy Y, Saad F. Is there a relationship between the severity of erectile dysfunction and the comorbidity profile in men with late onset hypogonadism? Arab J Urol 2015;13:162-8.

12. Li HJ, Kang DY. Prevalence of sexual dysfunction in men with chronic prostatitis/chronic pelvic pain syndrome: a metaanalysis. World J Urol 2016;34:1009-17.

13. Zhang Y, Zheng T, Tu X, Chen X, Wang Z, Chen S, et al. Erectile dysfunction in chronic prostatitis/chronic pelvic pain syndrome: outcomes from a multi-center study and risk factor analysis in a single center. PLoS One 2016;11:e0153054.

14. Morley JE, Charlton E, Patrick P, Kaiser FE, Cadeau P, McCready D, et al. Validation of a screening questionnaire for androgen deficiency in aging males. Metabolism 2000;49:1239-42.

15. Kang SY, Lee JA, Sunwoo S, Yu BY, Lee JH, Cho CH, et al. Prevalence of sexual dysfunction and associated risk factors in middle-aged and elderly Korean men in primary care. J Sex Res 2016;53:1165-78.

16. Tajar A, Huhtaniemi IT, O'Neill TW, Finn JD, Pye SR, Lee $\mathrm{DM}$, et al. Characteristics of androgen deficiency in late-onset hypogonadism: results from the European Male Aging Study (EMAS). J Clin Endocrinol Metab 2012;97:1508-16.

17. Huhtaniemi I. Late-onset hypogonadism: current concepts and controversies of pathogenesis, diagnosis and treatment. Asian J Androl 2014;16:192-202.

18. Lee JH, Lee SW. Testosterone and chronic prostatitis/chronic pelvic pain syndrome: a propensity score-matched analysis. J Sex Med 2016;13:1047-55.

19. Lee JH, Kim Y, Park YW, Lee DG. Relationship between benign prostatic hyperplasia/lower urinary tract symptoms and total serum testosterone level in healthy middle-aged eugonadal men. J Sex Med 2014;11:1309-15.

20. Tang WH, Zhuang XJ, Shu RM, Guan D, Ji YD, Zhang BL, et al. The prevalence of erectile dysfunction among subjects with late-onset hypogonadism: a population-based study in China. Int J Clin Exp Med 2015;8:13901-10.

21. Marberger M, Wilson TH, Rittmaster RS. Low serum testosterone levels are poor predictors of sexual dysfunction. BJU Int 2011;108:256-62.

22. Lee JH. Associations between premature ejaculation, lower urinary tract symptoms, and erectile dysfunction in middleaged Korean policemen. J Sex Med 2014;11:1512-8.

23. Jackson G, Betteridge J, Dean J, Eardley I, Hall R, Holdright $\mathrm{D}$, et al. A systematic approach to erectile dysfunction in the cardiovascular patient: a Consensus Statement: update 2002. Int J Clin Pract 2002;56:663-71.

24. Ogden CL, Carroll MD, Curtin LR, McDowell MA, Tabak CJ, Flegal KM. Prevalence of overweight and obesity in the United States, 1999-2004. JAMA 2006;295:1549-55.

25. Chitaley K, Kupelian V, Subak L, Wessells H. Diabetes, obesity and erectile dysfunction: field overview and research priorities. J Urol 2009;182:S45-50.

26. Corona G, Rastrelli G, Maggi M. Treatment of premature ejaculation and comorbid endocrine and metabolic disorders. In: Jannini EA, McMahon CG, Waldinger MD, editors. Premature ejaculation: from etiology to diagnosis and treatment. Milano: Springer; 2013;289-303.

27. Pontari MA, Ruggieri MR. Mechanisms in prostatitis/chronic pelvic pain syndrome. J Urol 2004;172:839-45.

28. Schultheiss D. Urogenital infections and male sexuality: effects on ejaculation and erection. Andrologia 2008;40:125-9.

29. Dimitrakov J, Joffe HV, Soldin SJ, Bolus R, Buffington CA, Nickel JC. Adrenocortical hormone abnormalities in men with chronic prostatitis/chronic pelvic pain syndrome. Urology 2008;71:261-6. 